\title{
Average Age of Information for a Multi-Source M/M/1 Queueing Model With Packet Management
}

\author{
Mohammad Moltafet and Markus Leinonen \\ Centre for Wireless Communications - Radio Technologies \\ University of Oulu, Finland \\ e-mail: \{mohammad.moltafet, markus.leinonen\}@oulu.fi
}

\author{
Marian Codreanu \\ Department of Science and Technology \\ Linköping University, Sweden \\ e-mail: marian.codreanu@liu.se
}

\begin{abstract}
We consider a status update system consisting of two independent sources, one server, and one sink. The packets of different sources are generated according to the Poisson process and the packets are served according to an exponentially distributed service time. We consider the following packet management policy. When the system is empty, any arriving packet immediately enters the server; when the server is busy, a packet of a source waiting in the queue is replaced if a new packet of the same source arrives. We derive the average age of information (AoI) of the considered $\mathrm{M} / \mathrm{M} / \mathbf{1}$ queueing model by using the stochastic hybrid systems (SHS) technique. Numerical results are provided to show the effectiveness of the proposed policy.

Index Terms- Information freshness, age of information (AoI), multi-source queueing model, stochastic hybrid systems (SHS).
\end{abstract}

\section{INTRODUCTION}

Freshness of the status information at a receiver is a key performance enabler in a wide range of applications of the upcoming wireless network generation such as Internet of things, and cyber physical applications [1], [2]. The age of information (AoI) was introduced as a destination-centric metric that characterizes the freshness of the status information [3], [4]. A status update packet contains the measured value of a monitored process and a time stamp representing the time when the sample was generated. Due to wireless channel access, channel errors, and fading, etc., communicating a status update packet through the network experiences a random delay. If at a time instant $t$, the most recently received status update packet contains the time stamp $U(t)$, AoI is defined as the random process $\Delta(t)=t-U(t)$. Thus, the AoI measures for each sensor the time elapsed since the last received status update packet was generated at the sensor. The average AoI is the most commonly used metric to evaluate the AoI [1]-[13].

The first queueing theoretic work on AoI is [7] where the authors derived the average AoI for a single-source M/M/1 first-come first-served (FCFS) queueing model. The average AoI for an M/M/1 last-come first-served (LCFS) queueing model with preemption was analyzed in [3]. The average AoI for different packet management policies in a singlesource $\mathrm{M} / \mathrm{M} / 1$ queueing model were derived in [8]. The work [11] was the first to investigate the average AoI in a multi-source setup. The authors of [11] derived the average AoI for a multi-source M/M/1 FCFS queueing model. The closed-form expressions for the average AoI and average peak AoI in a multi-source $\mathrm{M} / \mathrm{G} / 1 / 1$ preemptive queueing model were derived in [12]. The authors of [13] derived an exact expression for the average AoI for a multi-source M/M/1 FCFS queueing model and an approximate expression for the average AoI for a multi-source M/G/1 FCFS queueing model having a general service time distribution.

The most related work to our paper is [1], where the authors introduced a powerful technique based on stochastic hybrid systems (SHS) to evaluate the AoI. They considered a multisource queueing model in which the packets are generated according to the Poisson process and served according to an exponentially distributed service time. The authors derived the average AoI for two packet management policies: 1) LCFS with preemption under service (LCFS-S), and 2) LCFS with preemption only in waiting (LCFS-W). Under the LCFS-S policy, a new arriving packet preempts any packet that is currently under service (regardless of the source index). Under the LCFS-W policy, a new arriving packet replaces any older packet waiting in the queue (regardless of the source index); however, the new packet has to wait for any update packet that is currently under service to finish.

In this paper, we consider a status update system in which two independent sources generate packets according to the Poisson process and the packets are served according to an exponentially distributed service time. The packet management policy is as follows. When the system is empty, any arriving packet immediately enters the server. Differently from the policies studied in [1], when the server is busy, a packet of a source waiting in the queue is replaced only if a new packet of the same source arrives. We derive the average AoI of the considered queueing model by using the SHS technique.

\section{SySTEM MOdEL}

We consider a status update system consisting of two independent sources, one server, and one sink, as depicted in Fig. 1. Each source observes a random process at random time instants. The sink is interested in timely information about the status of these random processes. Status updates are transmitted as packets, containing the measured value of the monitored process and a time stamp representing the time when the sample was generated. We assume that the packets of sources 1 and 2 are generated according to the Poisson process with rates $\lambda_{1}$ and $\lambda_{2}$, respectively, and the packets are served according to an exponentially distributed service time 


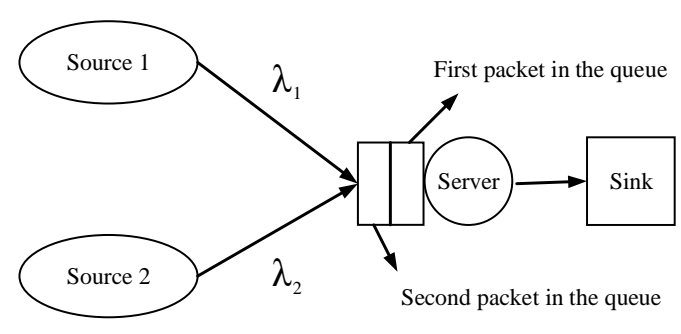

Fig. 1: The considered status update system.

with mean $1 / \mu$. Let $\rho_{1}=\lambda_{1} / \mu$ and $\rho_{2}=\lambda_{2} / \mu$ be the load of source 1 and 2 , respectively. Since packets of the sources are generated according to the Poisson process and the sources are independent, the packet generation in the system follows the Poisson process with rate $\lambda=\lambda_{1}+\lambda_{2}$. The overall load in the system is $\rho=\rho_{1}+\rho_{2}=\lambda / \mu$.

Packet management policy: The queue can contain at most two packets at the same time, one packet of source 1 and one packet of source 2. When the system is empty, any arriving packet immediately enters the server. When the server is busy, a packet of a source $c \in\{1,2\}$ waiting in the queue is replaced if a new packet of the same source arrives. The order of packets in the queue does not change based on new arrivals. In other words, when a new packet of a source $c \in\{1,2\}$ arrives, the only action is that the possible packet of the same source waiting in the queue is replaced by the fresh packet.

Definition 1 (AoI). Let $t_{c, i}$ denote the time instant at which the $i$ th status update packet of source $c$ was generated, and $t_{c, i}^{\prime}$ denote the time instant at which this packet arrives at the sink. At a time instant $\tau$, the index of the most recently received packet of source $c$ is given by $N_{c}(\tau)=\max \left\{i^{\prime} \mid t_{c, i^{\prime}}^{\prime} \leq \tau\right\}$, and the time stamp of the most recently received packet of source $c$ is $U_{c}(\tau)=t_{c, N_{c}(\tau)}$. The AoI of source $c$ at the destination is defined as the random process $\Delta_{c}(t)=t-U_{c}(t)$.

Let $(0, \tau)$ denote an observation interval. Accordingly, the time average AoI of the source $c$ at the sink, denoted as $\Delta_{\tau, c}$, is defined as $\Delta_{\tau, c}=\frac{1}{\tau} \int_{0}^{\tau} \Delta_{c}(t) \mathrm{d} t$. The average AoI of source $c$, denoted by $\Delta_{c}$, is defined as $\Delta_{c}=\lim _{\tau \rightarrow \infty} \Delta_{\tau, c}$.

\section{AoI Analysis Using the SHS Technique}

Next, we use the SHS technique introduced in [1], to calculate the average AoI of each source in the system. In the following, we briefly present the main idea behind the SHS technique. We refer the readers to [1] for more details.

\section{A. SHS Technique}

The SHS technique models a queueing system through the states $(q(t), \mathbf{x}(t))$, where $q(t) \in \mathcal{Q}=\{0,1, \cdots, m\}$ is a continuous-time finite-state Markov chain that describes the occupancy of the system and $\mathbf{x}(t)=\left[x_{0}(t) x_{1}(t) \cdots x_{n}(t)\right] \in \mathbb{R}^{1 \times(n+1)} \quad$ is a continuous process that describes the evolution of age-related processes at the sink. Following the approach in [1], we label the source of interest as source 1 and employ the continuous process $\mathbf{x}(t)$ to track the age of source 1 status updates at the sink.
The Markov chain $q(t)$ can be presented as a graph $(\mathcal{Q}, \mathcal{L})$ where each discrete state $q(t) \in \mathcal{Q}$ is a node of the chain and a (directed) link $l \in \mathcal{L}$ from node $q_{l}$ to node $q_{l}^{\prime}$ indicates a transition from state $q_{l} \in \mathcal{Q}$ to state $q_{l}^{\prime} \in \mathcal{Q}$. Note that a transition from a state to itself (i.e., a self-transition) is possible. Through a self-transition, a reset of the continuous state $\mathrm{x}$ takes place, but the discrete state remains the same (see [1, Section III]).

A transition occurs when a packet arrives or departs in the system. Since the time elapsed between departures and arrivals is exponentially distributed, the transition $l \in \mathcal{L}$ from state $q_{l}$ to state $q_{l}^{\prime}$ occurs with the exponential rate $\lambda^{(l)} \delta_{q_{l}, q(t)}$, where the Kronecker delta function $\delta_{q_{l}, q(t)}$ ensures that the transition $l$ occurs only when the discrete state $q(t)$ is equal to $q_{l}$. When a transition $l$ occurs, the discrete state $q_{l}$ jumps to state $q_{l}^{\prime}$, and the continuous state $\mathbf{x}$ is reset to $\mathbf{x}^{\prime}$ according to a binary transition reset map matrix $\mathbf{A}_{l} \in \mathbb{R}^{(n+1) \times(n+1)}$ as $\mathbf{x}^{\prime}=\mathbf{x} \mathbf{A}_{l}$. In addition, at each state $q(t)=q \in \mathcal{Q}$, the continuous state $\mathbf{x}$ evolves as a piece-wise linear function through the differential equation $\dot{\mathbf{x}}(t)=\frac{\partial \mathbf{x}(t)}{\partial t}=\mathbf{b}_{q}$, where $\mathbf{b}_{q}=\left[\begin{array}{llll}b_{q, 0} & b_{q, 1} \cdots b_{q, n}\end{array}\right]$ is a vector with binary elements, i.e., $b_{q, j} \in\{0,1\}, \forall j \in\{0, \cdots, n\}, q \in \mathcal{Q}$. If the age process $x_{j}(t)$ increases at a unit rate, we have $b_{q, j}=1$; otherwise, $b_{q, j}=0$.

To calculate the average AoI, the state probabilities of the Markov chain and the correlation vector between the discrete state $q(t)$ and the continuous state $\mathbf{x}(t)$ need to be calculated. Let $\pi_{q}(t)$ denote the probability of being in state $q$ and $\mathbf{v}_{q}(t)$ denote the correlation vector between the discrete state $q(t)$ and the continuous state $\mathbf{x}(t)$. Accordingly, we have

$$
\begin{gathered}
\pi_{q}(t)=\mathbb{E}\left[\delta_{q, q(t)}\right]=\operatorname{Pr}(q(t)=q), \\
\mathbf{v}_{q}(t)=\mathbb{E}\left[\mathbf{x}(t) \delta_{q, q(t)}\right]=\left[v_{q 0}(t) \cdots v_{q n}(t)\right] .
\end{gathered}
$$

Let $\mathcal{L}_{q}^{\prime}$ denote the set of incoming transitions and $\mathcal{L}_{q}$ the set of outgoing transitions for state $q$, defined as

$$
\mathcal{L}_{q}^{\prime}=\left\{l \in \mathcal{L}: q_{l}^{\prime}=q\right\}, \quad \mathcal{L}_{q}=\left\{l \in \mathcal{L}: q_{l}=q\right\} .
$$

Following the ergodicity assumption of the Markov chain $q(t)$ in the AoI analysis [1], [14], the state probability vector $\boldsymbol{\pi}(t)=\left[\pi_{0}(t) \cdots \pi_{m}(t)\right]$ converges uniquely to the stationary vector $\overline{\boldsymbol{\pi}}=\left[\bar{\pi}_{0} \cdots \bar{\pi}_{m}\right]$ satisfying [1]

$$
\begin{aligned}
& \bar{\pi}_{q} \sum_{l \in \mathcal{L}_{q}} \lambda^{(l)}=\sum_{l \in \mathcal{L}_{q}^{\prime}} \lambda^{(l)} \bar{\pi}_{q_{l}}, \quad \forall q \in \mathcal{Q}, \\
& \sum_{q \in \mathcal{Q}} \bar{\pi}_{q}=1 .
\end{aligned}
$$

As it has been shown in [1, Theorem 4], under the ergodicity assumption of the Markov chain $q(t)$, the correlation vector $\mathbf{v}_{q}(t)$ converges to a nonnegative limit $\overline{\mathbf{v}}_{q}=\left[\bar{v}_{q 0} \cdots \bar{v}_{q n}\right], \forall q \in$ $\mathcal{Q}$, as $t \rightarrow \infty$ such that

$$
\overline{\mathbf{v}}_{q} \sum_{l \in \mathcal{L}_{q}} \lambda^{(l)}=\mathbf{b}_{q} \bar{\pi}_{q}+\sum_{l \in \mathcal{L}_{q}^{\prime}} \lambda^{(l)} \overline{\mathbf{v}}_{q_{l}} \mathbf{A}_{l}, \quad \forall q \in \mathcal{Q} .
$$

The average AoI of source 1 is calculated by [1, Theorem 4]

$$
\Delta_{1}=\sum_{q \in \mathcal{Q}} \bar{v}_{q 0} .
$$

Thus, the main goal in deriving the average AoI is to calculate $\bar{v}_{q 0}, \forall q \in \mathcal{Q}$. 
TABLE I: SHS Markov chain states

\begin{tabular}{|c|l|l|l|}
\hline State & $\begin{array}{l}\text { Source index of } \\
\text { the second packet } \\
\text { in the queue }\end{array}$ & $\begin{array}{l}\text { Source index of } \\
\text { the first packet in } \\
\text { the queue }\end{array}$ & $\begin{array}{l}\text { Source index of } \\
\text { the packet under } \\
\text { service }\end{array}$ \\
\hline 0 & - & - & - \\
\hline 1 & - & - & 1 \\
\hline 2 & - & - & 2 \\
\hline 3 & - & 1 & 1 \\
\hline 4 & - & 2 & 1 \\
\hline 5 & 2 & 1 & 1 \\
\hline 6 & 1 & 2 & 1 \\
\hline 7 & - & 1 & 2 \\
\hline 8 & - & 2 & 2 \\
\hline 9 & 2 & 1 & 2 \\
\hline 10 & 1 & 2 & 2 \\
\hline
\end{tabular}

\section{B. Average AoI Calculation}

In our model, the state space of the Markov chain is $\mathcal{Q}=\{0,1, \cdots, 10\}$ as presented in Table I. For example, $q=0$ indicates that the server is idle, i.e., the system is empty; $q=1$ indicates that a source 1 packet is under service and the queue is empty; $q=5$ indicates that a source 1 packet is under service, the first packet in the queue (i.e., the packet that is ahead of the queue as depicted in Fig. 1) is a source 1 packet, and the second one in the queue is a source 2 packet.

In our queueing model, the continuous process is $\mathbf{x}(t)=\left[x_{0}(t) x_{1}(t) x_{2}(t) x_{3}(t)\right]$, where $x_{0}(t)$ is the current AoI of source 1 at time instant $t, \Delta_{1}(t) ; x_{1}(t)$ encodes what $\Delta_{1}(t)$ would become if the packet that is under service is delivered to the sink at time instant $t ; x_{2}(t)$ encodes what $\Delta_{1}(t)$ would become if the first packet in the queue is delivered to the sink at time instant $t ; x_{3}(t)$ encodes what $\Delta_{1}(t)$ would become if the second packet in the queue is delivered to the sink at time instant $t$. The transitions between the discrete states $q_{l} \rightarrow q_{l}^{\prime}, \forall l \in \mathcal{L}$, and their effects on the continuous state $\mathbf{x}(t)$ are summarized in Table II. In the following, we explain the transitions presented in Table II:

- $l=1$ : A source 1 packet arrives at an empty system. With this arrival/transition the AoI of source 1 does not change, i.e., $x_{0}^{\prime}=x_{0}$. This is because the arrival of source 1 packet does not yield an age reduction until it is delivered to the sink. Since the arriving source 1 packet is fresh and its age is zero, we have $x_{1}^{\prime}=0$. Since with this arrival the queue is still empty, $x_{2}$ and $x_{3}$ become irrelevant to the AoI of source 1 , and thus, $x_{2}^{\prime}=0$ and $x_{3}^{\prime}=0$. Finally, we have

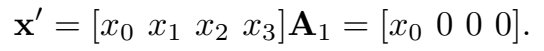

According to (7), it can be shown that $\mathbf{A}_{1}$ is given by

$$
\mathbf{A}_{1}=\left[\begin{array}{llll}
1 & 0 & 0 & 0 \\
0 & 0 & 0 & 0 \\
0 & 0 & 0 & 0 \\
0 & 0 & 0 & 0
\end{array}\right] .
$$

Then, by using (8), $\overline{\mathbf{v}}_{0} \mathbf{A}_{1}$ is calculated as

$$
\overline{\mathbf{v}}_{0} \mathbf{A}_{1}=\left[\begin{array}{llll}
v_{00} & v_{01} & v_{02} & v_{03}
\end{array}\right] \mathbf{A}_{1}=\left[\begin{array}{llll}
v_{00} & 0 & 0 & 0
\end{array}\right] .
$$

\begin{tabular}{|c|c|c|c|c|}
\hline$l$ & $q_{l} \rightarrow q_{l}^{\prime}$ & $\lambda^{(l)}$ & $\mathbf{x} \mathbf{A}_{l}$ & $\mathbf{v}_{q_{l}} \mathbf{A}_{l}$ \\
\hline 1 & $0 \rightarrow 1$ & $\lambda_{1}$ & {$\left[\begin{array}{llll}x_{0} & 0 & 0 & 0\end{array}\right]$} & {$\left[\begin{array}{llll}v_{00} & 0 & 0 & 0\end{array}\right]$} \\
\hline 2 & $0 \rightarrow 2$ & $\lambda_{2}$ & {$\left[\begin{array}{llll}x_{0} & 0 & 0 & 0\end{array}\right]$} & {$\left[\begin{array}{llll}v_{00} & 0 & 0 & 0\end{array}\right]$} \\
\hline 3 & $1 \rightarrow 3$ & $\lambda_{1}$ & {$\left[\begin{array}{llll}x_{0} & x_{1} & 0 & 0\end{array}\right]$} & {$\left[\begin{array}{llll}v_{10} & v_{11} & 0 & 0\end{array}\right]$} \\
\hline 4 & $1 \rightarrow 4$ & $\lambda_{2}$ & {$\left[\begin{array}{llll}x_{0} & x_{1} & 0 & 0\end{array}\right]$} & {$\left[\begin{array}{llll}v_{10} & v_{11} & 0 & 0\end{array}\right]$} \\
\hline 5 & $2 \rightarrow 7$ & $\lambda_{1}$ & {$\left[\begin{array}{llll}x_{0} & 0 & 0 & 0\end{array}\right]$} & {$\left[\begin{array}{llll}v_{20} & 0 & 0 & 0\end{array}\right]$} \\
\hline 6 & $2 \rightarrow 8$ & $\overline{\lambda_{2}}$ & $\left.\begin{array}{llll}x_{0} & 0 & 0 & 0\end{array}\right]$ & $\left.\begin{array}{lllll}v_{20} & 0 & 0 & 0\end{array}\right]$ \\
\hline 7 & $1 \rightarrow 0$ & $\mu$ & {$\left[\begin{array}{llll}x_{1} & 0 & 0 & 0\end{array}\right]$} & {$\left[\begin{array}{llll}v_{11} & 0 & 0 & 0\end{array}\right]$} \\
\hline 8 & $2 \rightarrow 0$ & $\mu$ & {$\left[\begin{array}{llll}x_{0} & 0 & 0 & 0\end{array}\right]$} & {$\left[\begin{array}{llll}v_{20} & 0 & 0 & 0\end{array}\right]$} \\
\hline 9 & $3 \rightarrow 3$ & $\lambda_{1}$ & {$\left[\begin{array}{llll}x_{0} & x_{1} & 0 & 0\end{array}\right]$} & {$\left[\begin{array}{llll}v_{30} & v_{31} & 0 & 0\end{array}\right]$} \\
\hline 10 & $4 \rightarrow 4$ & $\lambda_{2}$ & $\left.\begin{array}{llll}x_{0} & x_{1} & 0 & 0\end{array}\right]$ & {$\left[\begin{array}{llll}v_{40} & v_{41} & 0 & 0\end{array}\right]$} \\
\hline 11 & $3 \rightarrow 5$ & $\lambda_{2}$ & {$\left[\begin{array}{llll}x_{0} & x_{1} & x_{2} & 0\end{array}\right]$} & {$\left[\begin{array}{llll}v_{30} & v_{31} & v_{32} & 0\end{array}\right.$} \\
\hline 12 & $4 \rightarrow 6$ & $\lambda_{1}$ & {$\left[\begin{array}{llll}x_{0} & x_{1} & 0 & 0\end{array}\right]$} & {$\left[\begin{array}{llll}v_{40} & v_{41} & 0 & 0\end{array}\right]$} \\
\hline 13 & $5 \rightarrow 5$ & $\lambda_{1}$ & $\left.\begin{array}{llll}x_{0} & x_{1} & 0 & 0\end{array}\right]$ & {$\left[\begin{array}{llll}v_{50} & v_{51} & 0 & 0\end{array}\right]$} \\
\hline 14 & $5 \rightarrow 5$ & $\lambda_{2}$ & {$\left[\begin{array}{llll}x_{0} & x_{1} & x_{2} & 0\end{array}\right]$} & {$\left[\begin{array}{llll}v_{50} & v_{51} & v_{52} & 0\end{array}\right.$} \\
\hline 15 & $6 \rightarrow 6$ & $\lambda_{1}$ & {$\left[\begin{array}{llll}x_{0} & x_{1} & 0 & 0\end{array}\right]$} & {$\left[\begin{array}{llll}v_{60} & v_{61} & 0 & 0\end{array}\right]$} \\
\hline 16 & $6 \rightarrow 6$ & $\lambda_{2}$ & $\left.\begin{array}{llll}x_{0} & x_{1} & 0 & 0\end{array}\right]$ & $\left.\begin{array}{lllll}v_{60} & v_{61} & 0 & 0\end{array}\right]$ \\
\hline 17 & $7 \rightarrow 7$ & $\lambda_{1}$ & {$\left[\begin{array}{llll}x_{0} & 0 & 0 & 0\end{array}\right]$} & {$\left[\begin{array}{llll}v_{70} & 0 & 0 & 0\end{array}\right]$} \\
\hline 18 & $7 \rightarrow 9$ & $\lambda_{2}$ & {$\left[\begin{array}{llll}x_{0} & 0 & x_{2} & 0\end{array}\right]$} & {$\left[\begin{array}{llll}v_{70} & 0 & v_{72} & 0\end{array}\right]$} \\
\hline 19 & $8 \rightarrow 8$ & $\lambda_{2}$ & {$\left[\begin{array}{llll}x_{0} & 0 & 0 & 0\end{array}\right]$} & {$\left[\begin{array}{llll}v_{80} & 0 & 0 & 0\end{array}\right]$} \\
\hline 20 & $8 \rightarrow 10$ & $\lambda_{1}$ & {$\left[\begin{array}{llll}x_{0} & 0 & 0 & 0\end{array}\right]$} & {$\left[\begin{array}{llll}v_{80} & 0 & 0 & 0\end{array}\right]$} \\
\hline 21 & $9 \rightarrow 9$ & $\lambda_{1}$ & {$\left[\begin{array}{llll}x_{0} & 0 & 0 & 0\end{array}\right]$} & {$\left[\begin{array}{llll}v_{90} & 0 & 0 & 0\end{array}\right]$} \\
\hline 22 & $9 \rightarrow 9$ & $\lambda_{2}$ & {$\left[\begin{array}{llll}x_{0} & 0 & x_{2} & 0\end{array}\right]$} & {$\left[\begin{array}{llll}v_{90} & 0 & v_{92} & 0\end{array}\right]$} \\
\hline 23 & $10 \rightarrow 10$ & $\lambda_{1}$ & {$\left[\begin{array}{llll}x_{0} & 0 & 0 & 0\end{array}\right]$} & {$\left[\begin{array}{lllll}v_{100} & 0 & 0 & 0\end{array}\right]$} \\
\hline 24 & $10 \rightarrow 10$ & $\lambda_{2}$ & {$\left[\begin{array}{llll}x_{0} & 0 & 0 & x_{3}\end{array}\right]$} & {$\left[\begin{array}{llll}v_{100} & 0 & 0 & v_{103}\end{array}\right.$} \\
\hline 25 & $3 \rightarrow 1$ & $\mu$ & $\left.\begin{array}{llll}x_{1} & x_{2} & 0 & 0\end{array}\right]$ & {$\left[\begin{array}{llll}v_{31} & v_{32} & 0 & 0\end{array}\right]$} \\
\hline 26 & $4 \rightarrow 2$ & $\mu$ & {$\left[\begin{array}{llll}x_{1} & 0 & 0 & 0\end{array}\right]$} & {$\left[\begin{array}{llll}v_{41} & 0 & 0 & 0\end{array}\right]$} \\
\hline 27 & $5 \rightarrow 4$ & $\mu$ & {$\left[\begin{array}{llll}x_{1} & x_{2} & 0 & 0\end{array}\right]$} & {$\left[\begin{array}{llll}v_{51} & v_{52} & 0 & 0\end{array}\right]$} \\
\hline 28 & $6 \rightarrow 7$ & $\mu$ & {$\left[\begin{array}{llll}x_{1} & 0 & x_{3} & 0\end{array}\right]$} & {$\left[\begin{array}{llll}v_{61} & 0 & v_{63} & 0\end{array}\right]$} \\
\hline 29 & $7 \rightarrow 1$ & $\mu$ & $\left.\begin{array}{llll}x_{0} & x_{2} & 0 & 0\end{array}\right]$ & {$\left[\begin{array}{llll}v_{70} & v_{72} & 0 & 0\end{array}\right]$} \\
\hline 30 & $8 \rightarrow 2$ & $\mu$ & {$\left[\begin{array}{llll}x_{0} & 0 & 0 & 0\end{array}\right]$} & {$\left[\begin{array}{llll}v_{80} & 0 & 0 & 0\end{array}\right]$} \\
\hline 31 & $9 \rightarrow 4$ & $\mu$ & $\left.\begin{array}{llll}x_{0} & x_{2} & 0 & 0\end{array}\right]$ & {$\left[\begin{array}{llll}v_{90} & v_{92} & 0 & 0\end{array}\right]$} \\
\hline 32 & $10 \rightarrow 7$ & $\mu$ & {$\left[\begin{array}{llll}x_{0} & 0 & x_{3} & 0\end{array}\right]$} & {$\left[\begin{array}{llll}v_{100} & 0 & v_{103} & 0\end{array}\right.$} \\
\hline
\end{tabular}

TABLE II: Transition rates for the Markov chain

It can be seen from (7)-(9) that when we have $\mathbf{x}^{\prime}$ for a transition $l \in \mathcal{L}$, it is easy to calculate $\overline{\mathbf{v}}_{q_{l}} \mathbf{A}_{l}$. Thus, for the rest of the transitions, we just explain the calculation of $\mathbf{x}^{\prime}$ and present the final expression of $\overline{\mathbf{v}}_{q_{l}} \mathbf{A}_{l}$.

- $l=2$ : A source 2 packet arrives at an empty system. We have $x_{0}^{\prime}=x_{0}$, because this arrival does not change the AoI at the sink. Since the arriving packet is a source 2 packet, $x_{1}$ is irrelevant and we have $x_{1}^{\prime}=0$. Moreover, since the queue is empty, $x_{2}$ and $x_{3}$ become irrelevant, and we have $x_{2}^{\prime}=0$ and $x_{3}^{\prime}=0$.

- $l=3$ : A source 1 packet is under service and a source 1 packet arrives. In this transition, we have $x_{0}^{\prime}=x_{0}$ because there is no departure. The delivery of the packet under service reduces the AoI to $x_{1}$ and thus, $x_{1}^{\prime}=x_{1}$. Since the arriving source 1 packet is fresh and its age is zero we have $x_{2}^{\prime}=0$. Since there is only one packet in the queue, $x_{3}$ becomes irrelevant, and we have $x_{3}^{\prime}=0$. The reset map of transition $l=4$ can be derived similarly.

- $l=5$ : A source 2 packet is under service and a source 1 packet arrives. In this transition, we have $x_{0}^{\prime}=x_{0}$ because there is no departure. Since the packet under service is a source 2 packet, $x_{1}$ is irrelevant, and thus, $x_{1}^{\prime}=0$. Since the arriving source 1 packet is fresh and its age is zero, we have $x_{2}^{\prime}=0$. Since there is only one packet in the queue, we have $x_{3}^{\prime}=0$. The reset map of transition $l=6$ can be derived similarly.

- $l=7$ : A source 1 packet completes service and is delivered 
to the sink. With this transition, the AoI at the sink is reset to the age of the source 1 packet that just completed service, and thus, $x_{0}^{\prime}=x_{1}$. Since the system enters state $q=0$, we have $x_{1}^{\prime}=0, x_{2}^{\prime}=0$, and $x_{3}^{\prime}=0$. The reset map of transition $l=8$ can be derived similarly.

- $l=9$ : A source 1 packet is under service, a source 1 packet is in the queue, and a source 1 packet arrives. The source 1 packet in the queue is replaced by the fresh source 1 packet. In this transition, we have $x_{0}^{\prime}=x_{0}$ because there is no departure. The delivery of the packet under service reduces the AoI to $x_{1}$, and thus, $x_{1}^{\prime}=x_{1}$. Since the arriving source 1 packet is fresh and its age is zero, we have $x_{2}=0$. Since there is only one packet in the queue, we have $x_{3}^{\prime}=0$. The reset maps of transitions $l=10$, $l=17$, and $l=19$ can be derived similarly.

- $l=11$ : A source 1 packet is under service, a source 1 packet is in the queue, and a source 2 packet arrives. In this transition, $x_{0}^{\prime}=x_{0}$ because there is no departure. The delivery of the packet under service reduces the AoI to $x_{1}$, and thus, $x_{1}^{\prime}=x_{1}$. The delivery of the first packet in the queue reduces the AoI to $x_{2}$, and thus, $x_{2}^{\prime}=x_{2}$. Since the second packet in the queue is a source 2 packet, we have $x_{3}^{\prime}=0$. The reset maps of transitions $l=12$, $l=18$, and $l=20$ can be derived similarly.

- $l=13$ : A source 1 packet is under service, the first packet in the queue is a source 1 packet, the second packet in the queue is a source 2 packet, and a source 1 packet arrives. According to the packet management policy, the source 1 packet in the queue is replaced by the fresh source 1 packet. In this transition, we have $x_{0}^{\prime}=x_{0}$ because there is no departure. The delivery of the packet under service reduces the AoI to $x_{1}$, thus, $x_{1}^{\prime}=x_{1}$. Since the arriving source 1 packet is fresh and its age is zero we have $x_{2}^{\prime}=0$. Since the second packet in the queue is a source 2 packet, we have $x_{3}^{\prime}=0$. The reset maps of transitions $l=14, l=15, l=16, l=21, l=22, l=23$, and $l=24$ can be derived similarly.

- $l=25$ : A source 1 packet is in the queue, and the source 1 packet completes service and is delivered to the sink. With this transition, the AoI at the sink is reset to the age of the source 1 packet that just completed service, and thus, $x_{0}^{\prime}=x_{1}$. Since the source 1 packet in the queue goes to the server, we have $x_{1}^{\prime}=x_{2}$. In addition, since with this departure the queue becomes empty, we have $x_{2}^{\prime}=0$ and $x_{3}^{\prime}=0$. The reset maps of transitions $l=26$, $l=29$, and $l=30$ can be derived similarly.

- $l=27$ : The first packet in the queue is a source 1 packet, the second packet in the queue is a source 2 packet, and the source 1 packet under service completes service and is delivered to the sink. With this transition, the AoI at the sink is reset to the age of the source 1 packet that just completed service, and thus, $x_{0}^{\prime}=x_{1}$. Since the first packet in the queue goes to the server, we have $x_{1}^{\prime}=x_{2}$. In addition, since with this departure the queue holds one source 2 packet, $x_{2}$ and $x_{3}$ become irrelevant, and thus, $x_{2}^{\prime}=0$ and $x_{3}^{\prime}=0$. The reset maps of transitions $l=28$, $l=31$, and $l=32$ can be derived similarly.

Recall that our goal is to find $\bar{v}_{q 0}, \forall q \in \mathcal{Q}$, to calculate the average AoI of source 1 in (6). In this regard, first we determine $\mathbf{b}_{q}, \forall q \in \mathcal{Q}$, and the stationary probability vector $\overline{\boldsymbol{\pi}}$. Then, by solving the linear equations in (5), we calculate $\bar{v}_{q 0}, \forall q \in \mathcal{Q}$.

The evolution of $\mathbf{x}(t)$ at each discrete state $q(t)=q$ is determined by $\mathbf{b}_{q}$, i.e., $\dot{\mathbf{x}}=\mathbf{b}_{q}$. Thus, the first element of $\mathbf{b}_{q}$ is equal to 1 in all discrete states, $b_{q, 1}=1, \forall q \in \mathcal{Q}$. This is because the AoI of source $1, \Delta_{1}(t)=x_{0}(t)$, increases at a unit rate with time in all discrete states. The second element of $\mathbf{b}_{q}$ is equal to 1 if there is a relevant packet (i.e., a packet of source 1) under service at state $q(t)=q$. The third element of $\mathbf{b}_{q}$ is equal to 1 if the first packet in the queue is a relevant packet at state $q(t)=q$. The fourth element of $\mathbf{b}_{q}$ is equal to 1 if the second packet in the queue is a relevant packet at state $q(t)=q$. Thus, $\mathbf{b}_{q}$ for different states are determined by

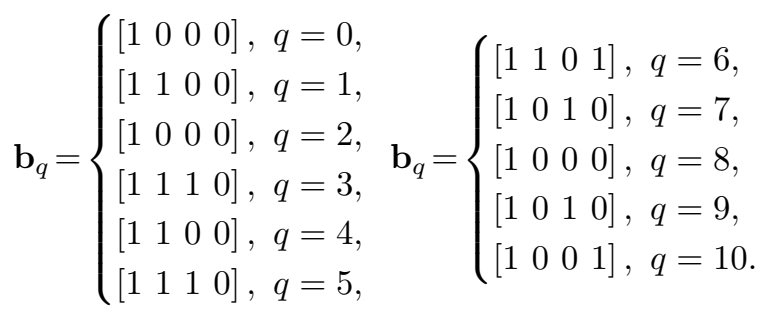

To calculate the stationary probabilities, we use (3) and (4). Using (3) and the transition rates among different states presented in Table II, it can be shown that the stationary probability vector $\overline{\boldsymbol{\pi}}$ satisfies $\overline{\boldsymbol{\pi}} \mathrm{D}=\overline{\boldsymbol{\pi}} \mathrm{Q}$ with

$$
\begin{aligned}
& \mathbf{D}= \operatorname{diag}[\lambda, \lambda+\mu, \lambda+\mu, \lambda+\mu, \lambda+\mu, \lambda+\mu, \lambda+\mu, \lambda+\mu, \\
&\lambda+\mu, \lambda+\mu, \lambda+\mu], \\
& \mathbf{Q}=\left[\begin{array}{ccccccccccc}
0 & \lambda_{1} & \lambda_{2} & 0 & 0 & 0 & 0 & 0 & 0 & 0 & 0 \\
\mu & 0 & 0 & \lambda_{1} & \lambda_{2} & 0 & 0 & 0 & 0 & 0 & 0 \\
\mu & 0 & 0 & 0 & 0 & 0 & 0 & \lambda_{1} & \lambda_{2} & 0 & 0 \\
0 & \mu & 0 & \lambda_{1} & 0 & \lambda_{2} & 0 & 0 & 0 & 0 & 0 \\
0 & 0 & \mu & 0 & \lambda_{2} & 0 & \lambda_{1} & 0 & 0 & 0 & 0 \\
0 & 0 & 0 & 0 & \mu & \lambda & 0 & 0 & 0 & 0 & 0 \\
0 & 0 & 0 & 0 & 0 & 0 & \lambda & \mu & 0 & 0 & 0 \\
0 & \mu & 0 & 0 & 0 & 0 & 0 & \lambda_{1} & 0 & \lambda_{2} & 0 \\
0 & 0 & \mu & 0 & 0 & 0 & 0 & 0 & \lambda_{2} & 0 & \lambda_{1} \\
0 & 0 & 0 & 0 & \mu & 0 & 0 & 0 & 0 & \lambda & 0 \\
0 & 0 & 0 & 0 & 0 & 0 & 0 & \mu & 0 & 0 & \lambda
\end{array}\right] .
\end{aligned}
$$

Applying (4), the stationary probabilities are given as

$$
\begin{aligned}
& \bar{\pi}_{0}=\frac{1}{\Phi}, \bar{\pi}_{1}=\frac{\rho_{1}}{\Phi}, \bar{\pi}_{2}=\frac{\rho_{2}}{\Phi}, \bar{\pi}_{3}=\frac{\rho_{1}^{2}}{\Phi}, \bar{\pi}_{4}=\frac{\rho_{1} \rho_{2}(1+\rho)}{\Phi\left(1+\rho_{1}\right)}, \\
& \bar{\pi}_{5}=\frac{\rho_{1}^{2} \rho_{2}}{\Phi\left(1+\rho_{2}\right)}, \bar{\pi}_{6}=\frac{\rho_{1}^{2} \rho_{2}(1+\rho)}{\Phi\left(1+\rho_{1}\right)}, \bar{\pi}_{7}=\frac{\rho_{1} \rho_{2}(1+\rho)}{\Phi\left(1+\rho_{2}\right)} \\
& \bar{\pi}_{8}=\frac{\rho_{2}^{2}}{\Phi\left(1+\rho_{1}\right)}, \bar{\pi}_{9}=\frac{\rho_{1} \rho_{2}^{2}(2+\rho)}{\Phi\left(1+\rho_{2}\right)}, \bar{\pi}_{10}=\frac{\rho_{1} \rho_{2}^{2}}{\Phi} .
\end{aligned}
$$

where $\Phi=\rho^{2}+\rho\left(2 \rho_{1} \rho_{2}+1\right)+1$.

By substituting (10) and (11) into (5) and solving the corresponding linear equations, the values of $\bar{v}_{q 0}, \forall q \in \mathcal{Q}$, are calculated. Finally, by substituting the results into (6), the 


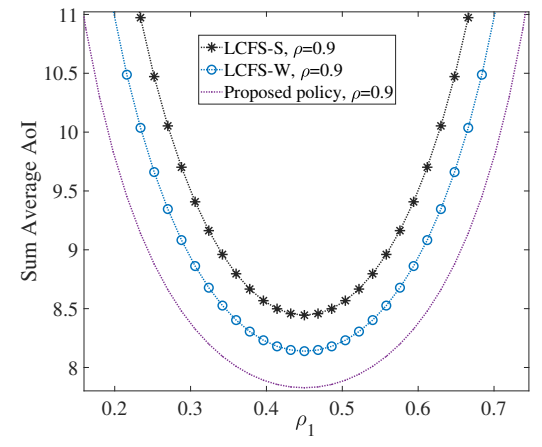

(a)

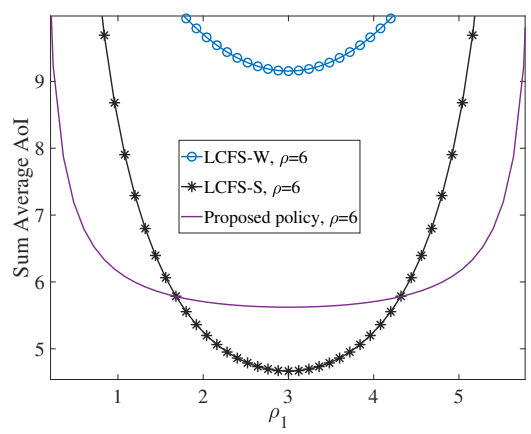

(b)

Fig. 2: Sum average AoI as a function of $\rho_{1}$ under different packet management policies for $\mu=1$ with (a) $\rho=0.9$, and (b) $\rho=6$.

average AoI of source 1 in the considered queueing model is given as

$$
\Delta_{1}=\frac{\sum_{i=0}^{7} \rho_{1}^{i} \psi_{i}}{\mu \rho_{1}\left(1+\rho_{1}\right)^{2}\left(\sum_{j=0}^{4} \rho_{1}^{j} \xi_{j}\right)},
$$

$\psi_{0}=\rho_{2}^{4}+2 \rho_{2}^{3}+3 \rho_{2}^{2}+2 \rho_{2}+1, \psi_{1}=7 \rho_{2}^{4}+15 \rho_{2}^{3}+21 \rho_{2}^{2}+14 \rho_{2}+6$, $\psi_{2}=17 \rho_{2}^{4}+46 \rho_{2}^{3}+64 \rho_{2}^{2}+42 \rho_{2}+16$,

$\psi_{3}=15 \rho_{2}^{4}+73 \rho_{2}^{3}+118 \rho_{2}^{2}+78 \rho_{2}+26$,

$\psi_{4}=5 \rho_{2}^{4}+52 \rho_{2}^{3}+124 \rho_{2}^{2}+102 \rho_{2}+30$,

$\psi_{5}=15 \rho_{2}^{3}+66 \rho_{2}^{2}+79 \rho_{2}+24$,

$\psi_{6}=15 \rho_{2}^{2}+31 \rho_{2}+11, \psi_{7}=5 \rho_{2}+2$,

$\xi_{0}=\rho_{2}^{4}+2 \rho_{2}^{3}+3 \rho_{2}^{2}+2 \rho_{2}+1, \quad \xi_{1}=2 \rho_{2}^{4}+6 \rho_{2}^{3}+9 \rho_{2}^{2}+7 \rho_{2}+3$,

$\xi_{2}=6 \rho_{2}^{3}+12 \rho_{2}^{2}+10 \rho_{2}+4, \quad \xi_{3}=6 \rho_{2}^{2}+8 \rho_{2}+3, \xi_{4}=2 \rho_{2}+1$.

\section{Numerical Results}

In this section, we show the effectiveness of the proposed packet management policy. Fig. 2 depicts sum average AoI as a function of $\rho_{1}$ under different packet management policies with $\mu=1$; in Fig. 2(a), $\rho=\rho_{1}+\rho_{2}=0.9$, and in Fig. 2(b), $\rho=\rho_{1}+\rho_{2}=6$. From this figure, we can observe that the best policy that achieves a low value of the sum average AoI depends on the system parameters. Note that when $\lambda_{2}=0$, the proposed policy and the LCFS-W policy coincide as expected.

\section{COnClusions}

We considered an $\mathrm{M} / \mathrm{M} / 1$ status update system consisting of two independent sources, one server, and one sink. We proposed a packet management policy in which when a new packet of a source arrives, the only action is that the possible packet of the same source waiting in the queue is replaced by the fresh packet. We derived the average AoI for each source using the SHS technique.

\section{ACKNOWLEDGEMENTS}

This research has been financially supported by the Infotech Oulu, the Academy of Finland (grant 323698), and Academy of Finland 6Genesis Flagship (grant 318927). M. Codreanu would like to acknowledge the support of the European Union's Horizon 2020 research and innovation programme under the Marie Skłodowska-Curie Grant Agreement No. 793402 (COMPRESS NETS). The work of M. Leinonen has also been financially supported in part by the Academy of Finland (grant 319485). M. Moltafet would like to acknowledge the support of Finnish Foundation for Technology Promotion and HPY Research Foundation.

\section{REFERENCES}

[1] R. D. Yates and S. K. Kaul, "The age of information: Real-time status updating by multiple sources," IEEE Trans. Inform. Theory, vol. 65, no. 3, pp. 1807-1827, Mar. 2019.

[2] A. Kosta, N. Pappas, and V. Angelakis, "Age of information: A new concept, metric, and tool," Foun. and Trends in Net., vol. 12, no. 3, pp. 162-259, 2017.

[3] S. K. Kaul, R. D. Yates, and M. Gruteser, "Status updates through queues," in Proc. Conf. Inform. Sciences Syst. (CISS), Princeton, NJ, USA, Mar. 21-23, 2012, pp. 1-6.

[4] S. Kaul, M. Gruteser, V. Rai, and J. Kenney, "Minimizing age of information in vehicular networks," in Proc. Commun. Society. Conf. on Sensor, Mesh and Ad Hoc Commun. and Net., Salt Lake City, UT, USA, Jun. 27-30, 2011, pp. 350-358.

[5] M. Moltafet, M. Leinonen, and M. Codreanu, "Worst case age of information in wireless sensor networks: A multi-access channel," IEEE Wireless Commun. Lett., vol. 9, no. 3, pp. 321-325, Mar. 2020.

[6] M. Moltafet, M. Leinonen, M. Codreanu, and N. Pappas, "Power minimization in wireless sensor networks with constrained AoI using stochastic optimization," in Proc. Annual Asilomar Conf. Signals, Syst., Comp., Pacific Grove, USA, Nov. 3-6, 2019. [Online]. https://arxiv.org/pdf/1912.02421v1.

[7] S. Kaul, R. Yates, and M. Gruteser, "Real-time status: How often should one update?" in Proc. IEEE Int. Conf. on Computer. Commun. (INFOCOM), Orlando, FL, USA, Mar. 25-30, 2012, pp. 2731-2735.

[8] M. Costa, M. Codreanu, and A. Ephremides, "On the age of information in status update systems with packet management," IEEE Trans. Inform. Theory, vol. 62, no. 4, pp. 1897-1910, Apr. 2016.

[9] Y. Inoue, H. Masuyama, T. Takine, and T. Tanaka, "The stationary distribution of the age of information in FCFS single-server queues,' in Proc. IEEE Int. Symp. Inform. Theory, Aachen, Germany, Jun. 2530, 2017, pp. 571-575.

[10] E. Najm and R. Nasser, "Age of information: The gamma awakening," in Proc. IEEE Int. Symp. Inform. Theory, Barcelona, Spain, Jul. 10-16, 2016, pp. 2574-2578.

[11] R. D. Yates and S. Kaul, "Real-time status updating: Multiple sources," in Proc. IEEE Int. Symp. Inform. Theory, Cambridge, MA, USA, Jul. 1-6, 2012, pp. 2666-2670.

[12] E. Najm and E. Telatar, "Status updates in a multi-stream M/G/1/1 preemptive queue," in Proc. IEEE Int. Conf. on Computer. Commun. (INFOCOM), Honolulu, HI, USA, Apr. 15-19, 2018, pp. 124-129.

[13] M. Moltafet, M. Leinonen, and M. Codreanu, "On the age of information in multi-source queueing models," Accepted, IEEE Trans. Commun, [Online]. https://arxiv.org/abs/1911.07029v2, 2020.

[14] A. Maatouk, M. Assaad, and A. Ephremides, "On the age of information in a CSMA environment," IEEE/ACM Trans. Net., Early Access 2020. 